\title{
Quantificação de nutrientes na precipitação em um plantio de Eucalyptus urophylla x Eucalyptus globulus subsp. maidenii, Eldorado do Sul, RS
}

\author{
Quantification of nutrients in the rainfall in a Eucalyptus urophylla $x$ \\ Eucalyptus globulus subsp. maidenii plantation, Eldorado do Sul, RS
}

\author{
Mirian Lago Valente ${ }^{1}$, Mauro Valdir Schumacher², \\ Robson Schaff Corrêa ${ }^{3}$, Elias Frank Araújo ${ }^{4}$ e Peter Trüby ${ }^{5}$
}

\begin{abstract}
Resumo
A precipitação pluvial constitui uma via importante da ciclagem biogeoquímica em ambientes com a atividade de silvicultura. Este estudo objetivou quantificar o aporte de nutrientes contidos na precipitação pluvial em uma plantação com o híbrido Eucalyptus urophylla x Eucalyptus globulus subsp. maidenii localizado no município de Eldorado do Sul (RS). Durante o período entre os meses de abril (2007) a fevereiro (2009),

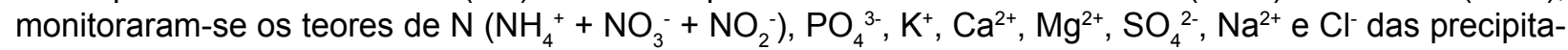
ções externa e interna. A interpretação dos resultados foi realizada por meio da análise de correlação de Pearson, onde se observou correlações entre a precipitação interna com os íons $\mathrm{K}^{+}, \mathrm{SO}_{4}{ }^{2-}, \mathrm{Na}^{2+}, \mathrm{Cl}^{-} \mathrm{e}$ com as frações do nitrogênio. A quantificação de nutrientes que retornou ao plantio via precipitação interna corresponderou a 5,$3 ; 6,8 ; 7,7 ; 16,7 ; 28,7 ; 44,4$ e $48,5 \mathrm{~kg} \mathrm{ha}^{-1}$ ano-1, respectivamente, para os nutrientes $\mathrm{Mg}$, $\mathrm{S}, \mathrm{N}, \mathrm{Ca}, \mathrm{K}, \mathrm{Na}$ e $\mathrm{Cl}$. Já para a precipitação externa, os valores observados corresponderam a 6,4; 11,8; 11,$3 ; 31,8 ; 15,4 ; 43,7$ e $49,4 \mathrm{~kg} \mathrm{ha}^{-1}$ ano-1 $^{-1}$ para os nutrientes $\mathrm{Mg}, \mathrm{S}, \mathrm{N}, \mathrm{Ca}, \mathrm{K}, \mathrm{Na}$ e $\mathrm{Cl}$, respectivamente. A contribuição da precipitação interna na quantidade total anual evidenciou a importância do ciclo nutricional dos elementos minerais para a manutenção sustentável do sítio florestal avaliado.
\end{abstract}

Palavras-chave: Ciclagem de nutrientes, silvicultura, precipitação interna; macronutrientes, micronutrientes.

\begin{abstract}
Rainfall is an important way of biogeochemical cycling in environments with forestry activity. This study aimed to quantify the nutrient input contained in the rainfall in a plantation with the hybrid Eucalyptus urophylla $x$ Eucalyptus globulus subsp. maidenii located in the county of Eldorado do Sul (RS). During

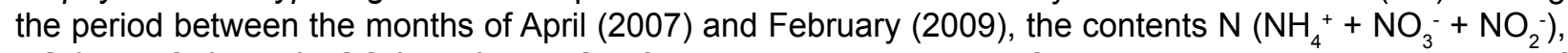
$\mathrm{PO}_{4}{ }^{3-}, \mathrm{K}^{+}, \mathrm{Ca}^{2+}, \mathrm{Mg}^{2+}, \mathrm{SO}_{4}{ }^{2-}, \mathrm{Na}^{2+}$ and $\mathrm{Cl}$ of internal and external rainfall were monitored. Interpretation of the results was performed using the Pearson correlation analysis, which observed correlations between the internal rainfall with ions $\mathrm{K}^{+}, \mathrm{SO}_{4}^{2-}, \mathrm{Na}^{2+}, \mathrm{Cl}^{-}$and the nitrogen fractions. The quantification of nutrients returned to planting by the internal rainfall corresponded to 5,$3 ; 6,8 ; 7,7 ; 16,7 ; 28,7 ; 44,4$ and $48,5 \mathrm{~kg} \mathrm{ha}^{-1}$ year $^{-1}$, respectively, for the nutrients $\mathrm{Mg}, \mathrm{S}, \mathrm{N}, \mathrm{Ca}, \mathrm{K}, \mathrm{Na}$ and $\mathrm{Cl}$. As for the rainfall, the observed values corresponded to 6,$4 ; 11,8 ; 11,3 ; 31,8 ; 15,4 ; 43,7$ and $49,4 \mathrm{~kg} \mathrm{ha}^{-1}$ year $^{-1}$ for nutrients $\mathrm{Mg}, \mathrm{S}, \mathrm{N}, \mathrm{Ca}, \mathrm{K}, \mathrm{Na}$ and $\mathrm{Cl}$, respectively. The contribution of internal rainfall in total annual amount emphasized the importance of the nutritional cycle of mineral elements for the sustainable maintenance of the evaluated forest site.
\end{abstract}

Keywords: Nutrient cycling, forestry, internal rainfall, macronutrients, micronutrients.

\section{INTRODUÇÃO}

As deposições atmosféricas constituem um dos principais mecanismos da ciclagem biogeoquímica e redistribuição dos vários nutrientes em ecossistemas florestais. A principal função desse mecanismo está relacionada com as superfícies dos galhos e folhas que agem como eficientes filtros,

${ }^{1}$ Doutoranda em Engenharia Florestal. UFSM - Universidade Federal de Santa Maria / Departamento de Solos. Avenida Roraima, 1000 - Camobi - 97105970 - Santa Maria, RS, Brasil. E-mail: mirian sm@yahoo.com.br

2Professor Titular do Departamento de Ciências Florestais. UFSM - Universidade Federal de Santa Maria. Avenida Roraima, 1000 - Camobi - 97105970 - Santa Maria, RS, Brasil. E-mail: mauro.schumacher@ufsm.br

3Professor Adjunto do Curso de Engenharia Florestal. UFG - Universidade Federal de Goiás, / Campus Jataí. Rod BR 364, km 192, 1800 - 75801615 - Jataí, GO - Brasil. E-mail: schaffcorrea@ufg.br

${ }^{4}$ Pesquisador Mestre. CMPC Celulose Rio Grandense. Rua São Geraldo, 1680 - 92500-000 - Guaiba, RS, Brasil. E-mail: efaraujo@cmpcrs.com.br

${ }^{5}$ Professor e pesquisador da Universidade de Freiburg. Universidade de Freiburg. Fahnenbergplatz, 79085 Freiburg im Breisgau, Alemanha. E-mail: peter.trueby@bodenkunde.uni-freiburg.de

Sci. For., Piracicaba, v. 44, n. 109, p. 249-259, mar. 2016 DOI: dx.doi.org/10.18671/scifor.v44n109.24 
retendo partículas e gases atmosféricos que são absorvidos pelas folhas ou aportados ao solo por meio da pluviosidade.

De acordo com Souza e Marques (2010), a ciclagem de nutrientes constitui um importante parâmetro na sustentabilidade florestal, sendo que dentre os fluxos de entrada e saída que interferem nesse processo, a pluviosidade que atinge a cobertura florestal contribui significativamente para a nutrição e manutenção do sítio florestal.

O maior desafio da silvicultura, hoje, constitui-se na produção sustentável de madeira, visto que a rápida taxa de crescimento dessas espécies eleva a demanda sobre os recursos do solo, principalmente, por água e nutrientes (BELLOTE et al., 2008).

As águas da chuva ao incidirem sobre a cobertura florestal trazem consigo elementos de constituição mineral e orgânica que se encontram suspensos na atmosfera e, ao atravessar o dossel florestal podem ser absorvidos, resultando numa importante fonte de reposição de nutrientes ao solo.

Este processo é considerado um dos mais importantes meios de entrada de nutrientes no ecossistema (HAAG, 1985; ARCOVA; CICCO, 1987). Com isso, o conhecimento da dinâmica biogeoquímica que ocorre em povoamentos com espécies de crescimento rápido permite avaliar os processos que são responsáveis pela manutenção da fertilidade do solo e da sustentabilidade da produção.

Os nutrientes da água da chuva geralmente estão prontamente disponíveis, enquanto que os da serapilheira são liberados gradualmente pela decomposição (SCHEER, 2009), o que torna essa fonte nutricional ainda mais importante quando as demais são escassas. Nesse contexto, vários estudos indicam um padrão de enriquecimento da água da chuva após a passagem da mesma pelo dossel florestal (LIMA, 1986; ARCOVA; CICCO, 1987; LOPES, 2001; LIU et al., 2002; SCHEER, 2006; ZHANG, et al. 2006; SCHEER, 2009).

Entretanto, ainda é incipiente a abordagem do quanto o aporte de nutrientes via precipitação pluvial contribui para a manutenção da fertilidade e adubação do sítio florestal e, consequentemente, para a sustentabilidade ecológica da atividade silvicultural.

Diante deste contexto, objetivou-se quantificar o aporte dos nutrientes $\mathrm{N}, \mathrm{P}, \mathrm{K}, \mathrm{Ca}, \mathrm{Mg}, \mathrm{S}, \mathrm{Na}$ e $\mathrm{Cl}$ contidos na precipitação pluvial numa plantação com híbrido Eucalyptus urophylla x Eucalyptus globulus subsp. maidenii localizado no município de Eldorado do Sul, RS.

\section{MATERIAL E MÉTODOS}

\section{Área de estudo}

\section{Localização e clima}

O presente estudo foi realizado em um povoamento florestal com o híbrido Eucalyptus urophylla x Eucalyptus globulus subsp. maidenii implantado durante o mês de julho de 2001 no Horto Florestal Terra Dura, com 41 ha. A Figura 1 apresenta a localização da área no município de Eldorado do Sul (RS), a uma altitude média de 154 m, em área pertence à Empresa Celulose Riograndense (Grupo CMPC).

O clima da região é classificado como subtemperado úmido com temperatura média anual igual a $18,6^{\circ} \mathrm{C}$ e precipitação média anual de $1.574 \mathrm{~mm}$ (MALUF, 2000). O solo da área é classificado como Argissolo Vermelho Distrófico abrúptico (CURI et al., 2009), onde os mesmos autores realizaram as análises químicas e granulométricas do solo no local de estudo, conforme descrito na Tabela 1.

O preparo do solo foi realizado por meio da subsolagem. Como adubação, aplicou-se $400 \mathrm{~kg}$ ha $^{-1}$ de fosfato reativo gafsa ( $28 \%$ de $\mathrm{P}_{2} \mathrm{O}_{5}$ total com $10 \%$ solúvel em ácido cítrico) no sulco e 100 $\mathrm{g}$ planta $^{-1}$ de NPK 06-30-06. Para a adubação de cobertura foram aplicados $150 \mathrm{~g} \mathrm{planta}^{-1}$ de NPK 15-5-30 aos 03 e aos 12 meses de idade das plantas. 
Tabela 1. Análise granulométrica e características químicas do solo da área de estudo, Eldorado do Sul (RS).

Table 1. Particle size analysis and chemical characteristics of the soil of the study area, Eldorado do Sul (RS).

\begin{tabular}{|c|c|c|c|c|c|c|c|c|c|c|c|c|}
\hline \multirow[t]{2}{*}{ Horizonte } & \multirow[t]{2}{*}{ Prof. (cm) } & $\begin{array}{c}\text { Areia } \\
\text { grossa }\end{array}$ & $\begin{array}{c}\text { Areia } \\
\text { fina }\end{array}$ & Silte & Argila & SB & $\mathbf{T}$ & $\mathrm{Ca}$ & Mg & $\mathbf{K}$ & $\mathrm{Na}$ & $\mathbf{N}$ \\
\hline & & \multicolumn{4}{|c|}{$--\%$} & \multicolumn{4}{|c|}{-- cmolc dm $^{-3}$-- } & \multicolumn{2}{|c|}{$\mathrm{mg} \mathrm{dm}^{-3}$} & total $(\%)$ \\
\hline$A p$ & $0-23$ & 32 & 16 & 17 & 35 & 2,1 & 10 & 1,2 & 0,7 & 47 & 10 & 1,8 \\
\hline BA & $23-36$ & 15 & 8 & 15 & 62 & 0,8 & 17,9 & 0,2 & 0,5 & 31 & 13 & 1,2 \\
\hline Bt1 & $36-77$ & 17 & 6 & 11 & 66 & 0,8 & 17,9 & 0,1 & 0,5 & 58 & 9,2 & 0,8 \\
\hline $\mathrm{Bt} 2$ & $77-187+$ & 18 & 7 & 15 & 60 & 0,8 & 17,9 & 0,1 & 0,6 & 30 & 10 & 0,8 \\
\hline \multirow{2}{*}{ Horizonte } & \multirow{2}{*}{ Prof. (cm) } & $\mathrm{pH}$ & $\mathbf{m}$ & $\mathbf{V}$ & $\mathbf{P}$ & B & $\mathbf{S}$ & Mn & $\mathrm{Cu}$ & $\mathrm{Zn}$ & $\mathrm{Fe}$ & C org \\
\hline & & $\mathrm{CaCl}_{2}$ & \multicolumn{2}{|c|}{$\%$} & \multicolumn{7}{|c|}{-- $\mathrm{mg} \mathrm{dm}^{-3}$-- } & $\mathrm{g} \mathrm{kg}^{-1}$ \\
\hline$A p$ & $0-23$ & 3.8 & 45 & 20,7 & 1,7 & 0,4 & 9,3 & 12,0 & 1,6 & 1,1 & 120,5 & 14,5 \\
\hline BA & $23-36$ & 3.8 & 81 & 4,7 & 0,9 & 0,2 & 19,5 & 0,7 & 0,9 & 0,6 & 22,0 & 8,7 \\
\hline Bt1 & $36-77$ & 3.8 & 83 & 4,6 & 0,6 & 0,2 & 47,3 & 1,0 & 0,2 & 0,4 & 3,0 & 3,5 \\
\hline $\mathrm{Bt} 2$ & $77-187+$ & 3.8 & 81 & 4,7 & 0,9 & 0,2 & 32,6 & 0,5 & 0,1 & 0,3 & 1,0 & 0,6 \\
\hline
\end{tabular}

Nota: SB- saturação de bases; $\mathrm{T}$ - capacidade de troca catiônica total a pH 0,7; $\mathrm{Ca}$ - cálcio; $\mathrm{Mg}$ - magnésio; $\mathrm{K}$ - potássio; $\mathrm{Na}$ - sódio; $\mathrm{N}$ - nitrogênio; $\mathrm{pH}$ : pH em CaCl$; \mathrm{m}$ - saturação por alumínio; $\mathrm{V}$ - saturação por base; $\mathrm{P}$ - fósforo; $\mathrm{B}$ - boro; $\mathrm{S}$ - enxofre; $\mathrm{Mn}$ - manganês; $\mathrm{Cu}$ - cobre; $\mathrm{Zn}$ - zinco; $\mathrm{Fe}$ - ferro; C org. - carbono orgânico.
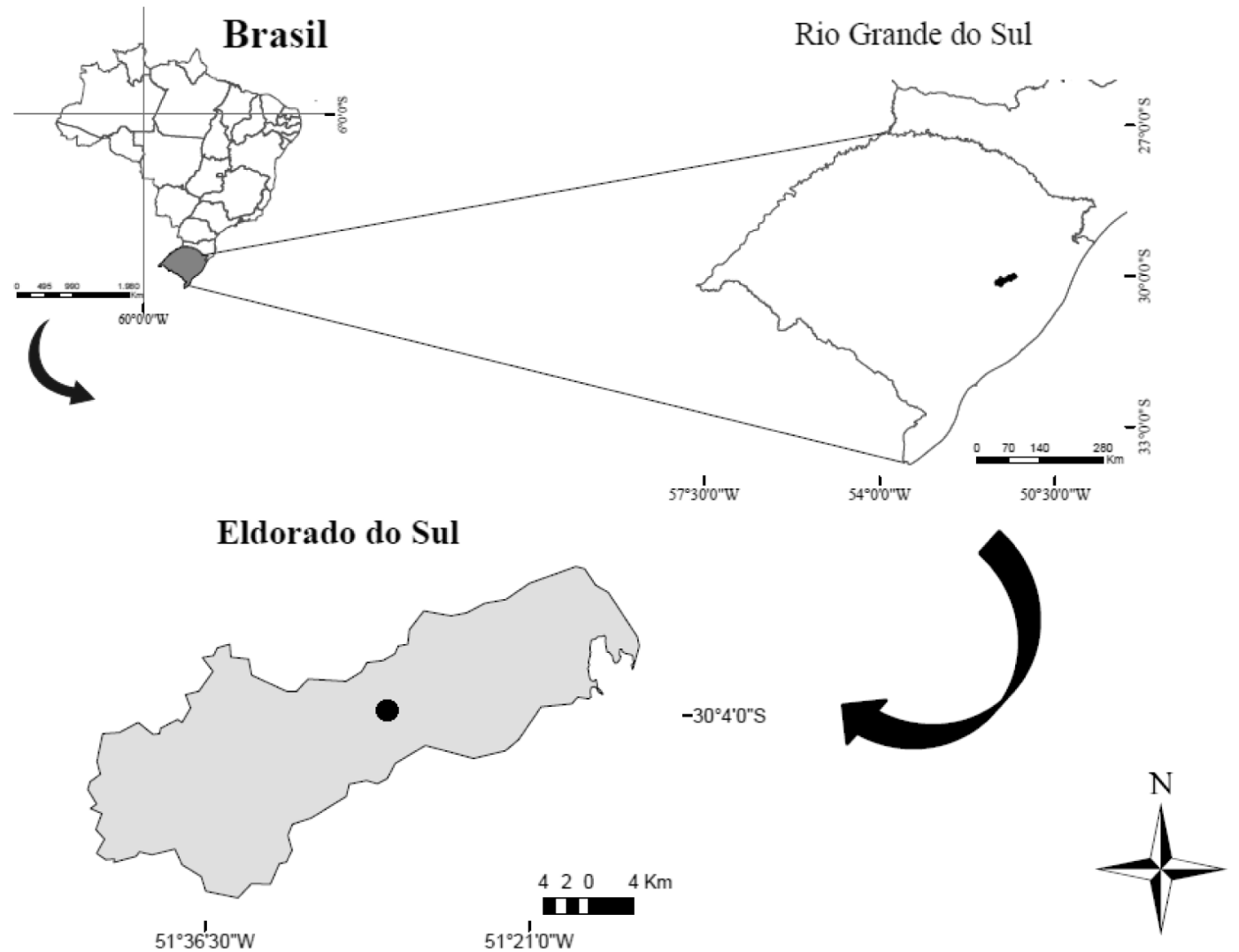

Figura 1. Localização da área experimental no município de Eldorado do Sul, RS.

Figure 1. Location of the experimental area in Eldorado do Sul, RS.

\section{Amostragem e quantificação dos nutrientes}

A avaliação do aporte de nutrientes via precipitação pluvial foi realizada por meio de funis coletores de água da chuva no interior do povoamento e na área de clareira. Para a estimativa da precipitação externa (PE) foram instalados 4 pluviômetros de polipropileno com $20 \mathrm{~cm}$ de diâmetro interno cada em área de clareira adjacente. Na borda dos pluviômetros foram anexadas agulhas com o objetivo de repelir os pássaros, que utilizam os funis como poleiros, contaminando a água coletada.

Para a avaliação da precipitação interna (PI) do povoamento, foram instaladas 4 parcelas de 20 $\mathrm{m}$ x $20 \mathrm{~m}$, onde foram alocados 3 pluviômetros por parcela idênticos aos da área de clareira, sendo um na linha de plantio, outro entre as linhas e o outro na diagonal entrelinhas, totalizando 12 pluviômetros no povoamento. 
As coletas foram efetuadas quinzenalmente entre abril de 2007 e fevereiro de 2009 (povoamento entre 6 e 8 anos, aproximadamente), resultando em 43 amostragens. Parte do conteúdo coletado foi armazenado em recipientes plásticos com capacidade de 0,5 litros cada. Após cada coleta, os pluviômetros foram lavados com água destilada e as amostras foram congeladas e enviadas para o Laboratório de Ecologia Florestal do Departamento de Ciências Florestais da UFSM para determinação das concentrações de $\mathrm{N}$ total $\left(\mathrm{NH}_{4}^{+}+\mathrm{NO}_{3}{ }^{-}+\mathrm{NO}_{2}{ }^{-}\right), \mathrm{PO}_{4}{ }^{3-}, \mathrm{K}^{+}, \mathrm{Ca}^{2+}, \mathrm{Mg}^{2+}, \mathrm{SO}_{4}{ }^{2-}, \mathrm{Na}^{2+}$ e $\mathrm{Cl}$. As amostras não continham a ação de soluções para controle da atividade biológica, como usado por Casartelli et al. (2008), optando-se por não usar estas substâncias, como em Juknys et al. (2007); Laclau et al. (2003).

\section{Análises químicas das pluviosidades}

As amostras de água foram analisadas conforme a metodologia do Standard methods for the examination of water and wastewater (APHA, 1998), com pré-tratamento de filtração simples (poros de $0,45 \mu \mathrm{m}$ ). Os teores foram determinados por cromatografia iônica sendo, os cátions, analisados por meio de colunas de cátions e os ânions por coluna de ânions.

\section{Análise estatística}

Primeiramente, realizou-se o tratamento dos dados brutos, como a verificação da presença de valores ausentes e outliers. Com o objetivo de avaliar o aporte nutricional em função das diferentes precipitações monitoradas (PI e PE), os teores dos cátions, ânions e a quantidade de precipitação pluvial para cada coleta, os dados foram submetidos a análise de Correlação de Pearson por meio do software SPSS 17.0.

\section{RESULTADOS E DISCUSSÃO}

A precipitação externa (PE) média foi de $1.705,5 \mathrm{~mm}$, valor um pouco acima da média histórica de $1.574 \mathrm{~mm}$ registrada por Maluf (2000). As médias mensais foram de $148 \mathrm{~mm}$ para a PE e de 130 mm para a PI, o que resulta numa interceptação média de $12 \%$.

Para os teores dos íons, o monitoramento dos diferentes elementos apresentou uma sazonalidade semelhante entre as precipitações analisadas (Figuras 2 e 3). Durante o período avaliado, teores de $\mathrm{PO}_{4}^{3-}$ não foram detectados ou quando detectados ficaram no limite da faixa de detecção dos aparelhos na maioria das análises, portanto não foram considerados nas discussões. Em geral, maiores concentrações foram verificadas na PI para os elementos $\mathrm{Cl}, \mathrm{Na}, \mathrm{K}, \mathrm{N}$ e $\mathrm{Mg}$ ocorrendo, principalmente, durante os meses de menor pluviosidade, o que evidencia uma maior deposição de aerossóis, poeira e outros materiais particulados sobre o dossel florestal nestas condições. Lima (1985) e Lopes (2013), em ecossistemas florestais, também observaram maiores concentrações de nutrientes na água das primeiras chuvas da estação, ou seja, após um longo período de estiagem. O mesmo também foi registrado por Pehl e Ray (1984), Allen et al. (1968), Meguro et al. (1979) e Arcova et al. (1993). Já para os períodos com maior pluviosidade (superiores a $100 \mathrm{~mm}$ ) se verificou menores valores dos teores, o que pode estar relacionado à diluição dos aerossóis oriundos da atmosfera (Figura 2 e 3 ).

Estas informações são pertinentes para o entendimento dos processos geoquímicos que podem vir a alterar a fertilidade do solo. A compreensão do aporte de nutrientes pode auxiliar no aperfeiçoamento das técnicas silviculturais relacionadas à manutenção da fertilidade do solo e sustentabilidade dos processos ecológicos.

Outro aspecto a considerar refere-se as alterações nos padrões de precipitação que podem ocorrer. Silva et al. (2014) relatam que para o Estado do Rio de Janeiro ocorrerão entre 2041 e 2070 eventos de precipitação com concentração do volume em eventos de curto período, bem como alterações no volume da precipitação, variando de $-350 \mathrm{~mm}$ a $300 \mathrm{~mm}$ dependendo da porção do território. Como a variação deste padrão é esperada para todo o país deverão ocorrer variações também nas concentrações dos íons ao longo do ano. Assim, poderão ocorrer alterações para as recomendações de adubação para eucalipto no Rio Grande do Sul. 


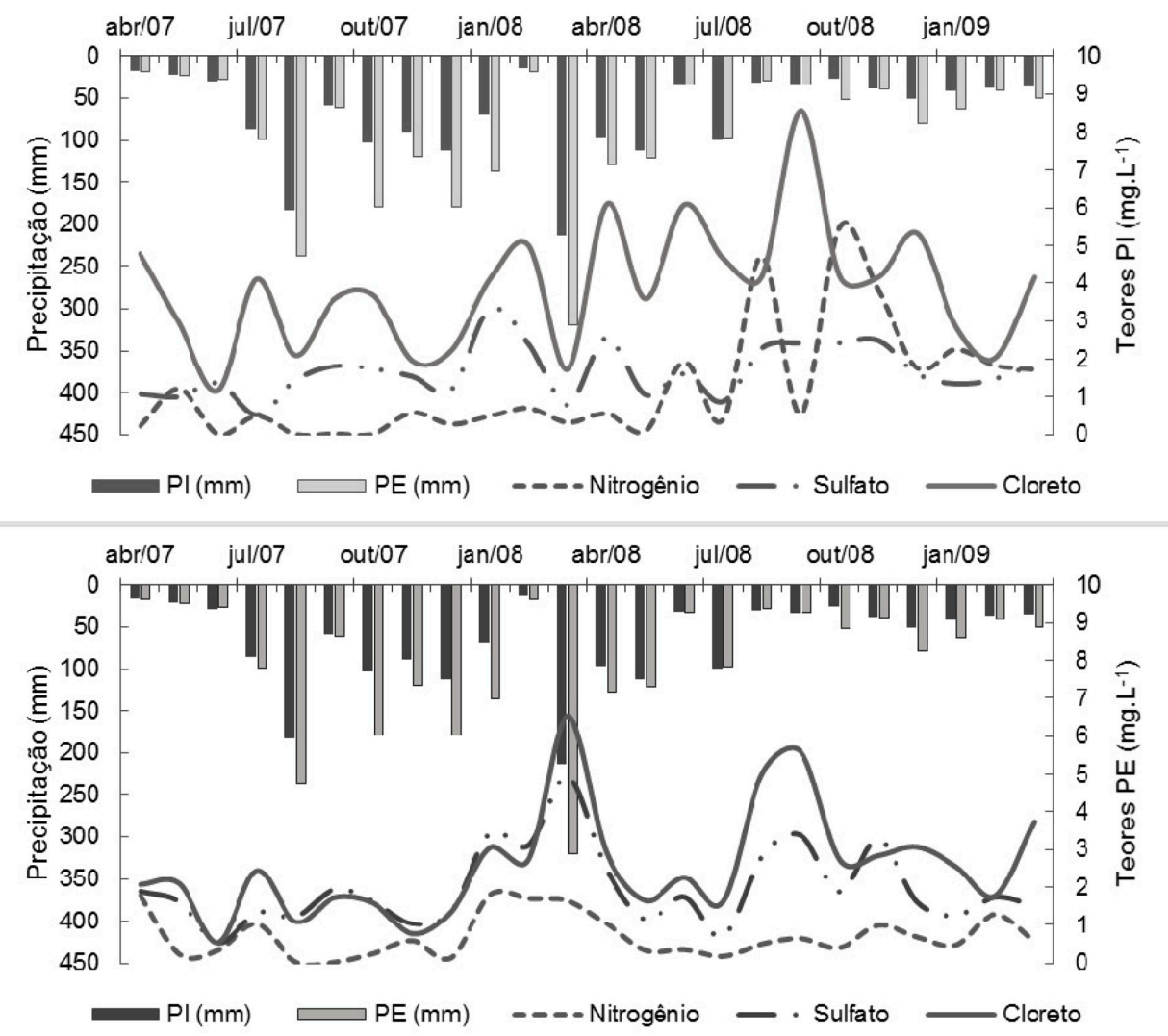

Figura 2. Concentrações de ânions $\left(\mathrm{mg} \mathrm{L}^{-1}\right)$ nas precipitações interna (PI) e externa (PE) durante período de abril de 2007 a fevereiro de 2009, Eldorado do Sul (RS).

Figure 2. Anion concentrations $\left(\mathrm{mg} \mathrm{L}^{-1}\right)$ in the internal rainfall (PI) and rainfall (PE) during the period from April 2007 to February 2009, Eldorado do Sul (RS).

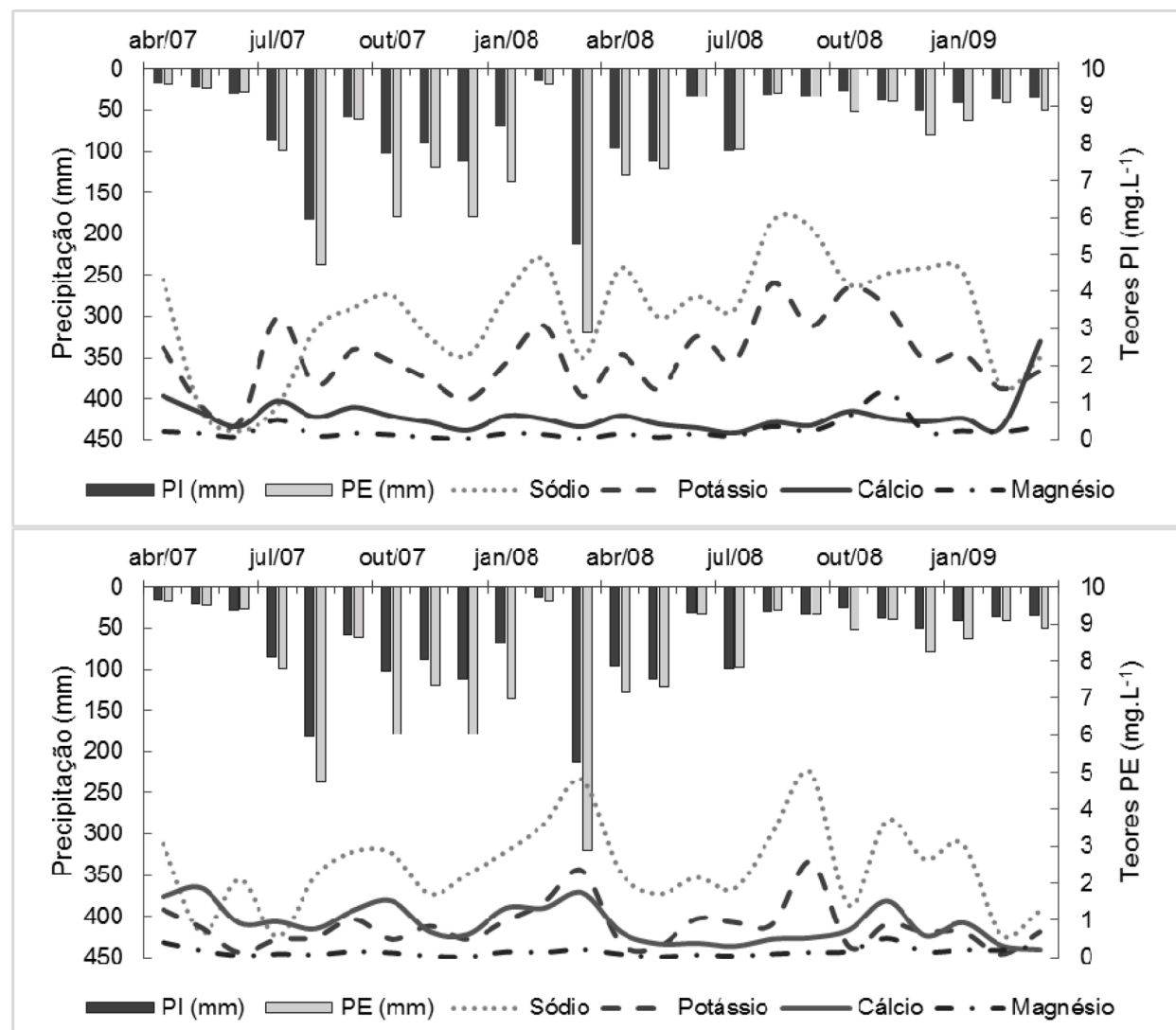

Figura 3. Concentrações de cátions $\left(\mathrm{mg} \mathrm{L}^{-1}\right)$ nas precipitações interna (PI) e externa (PE) durante período de abril de 2007 a fevereiro de 2009, Eldorado do Sul (RS).

Figure 3. Cations concentrations $\left(\mathrm{mg} \mathrm{L}^{-1}\right)$ in the internal rainfall (PI) and rainfall (PE) during the period from April 2007 to February 2009, Eldorado do Sul (RS). 
Além disso, as trajetórias das massas de ar de longas distâncias também podem atuar como fontes injetoras de íons no local de estudo. Segundo Freitas et al. (2000), o transporte a longa distância de partículas de aerossol segue o mesmo padrão de circulação atmosférica, fazendo com que as emissões da floresta amazônica saiam do continente Sul Americano por duas vias principais: o oceano Pacífico tropical e o oceano Atlântico Sul. A maior fonte de aerossóis para a atmosfera na América do Sul são as emissões por queimadas de florestas e cerrados, onde áreas com queimadas de 5 a 8 milhões de $\mathrm{km}^{2}$ são bastante comuns durante os meses de agosto, setembro e outubro (ARTAXO et al., 2006). Assim, os aerossóis provenientes das queimadas também poderiam influenciar nos povoamentos de eucalipto no Sul do Brasil, caso as massas de ar da primavera e começo do verão (trajetórias de massas de ar) continuem no sentido atual (Amazônia - Rio Grande do Sul).

Nas Tabelas 2 e 3, verificam-se os testes de correlação de Pearson entre os nutrientes e as precipitações monitoradas (PE e PI). Correlações significativas e negativas foram verificadas entre a PI e os íons $\mathrm{N}, \mathrm{SO}_{4}{ }^{2-}, \mathrm{Cl}^{-}, \mathrm{Na}^{2+}$ e $\mathrm{K}^{+}$(Tabela 3 ). A correlação negativa entre os parâmetros pode estar relacionada com o efeito de diluição dos íons em função dos volumes precipitados (LACLAU et al., 2003; CALIL, 2008), conforme já abordado anteriormente. Ao contrário do que alguns trabalhos da literatura evidenciaram (ZHANG et al., 2006; BALIEIRO et al., 2007), não ocorreu correlação positiva entre os teores de potássio e a PI (Tabela 3), talvez pelo efeito de diluição do íon nas amostras ao invés do processo de lixiviação em função da chuva. Scheer (2009) também verificou correlações negativas entre os teores de $\mathrm{K}^{+}, \mathrm{Ca}^{2+} \mathrm{e} \mathrm{Mg}^{2+}$ com a PI em uma Floresta Ombrófila Densa, em Guaraqueçaba (PR), o que pode indicar que o volume de chuva rege as quantidades de elementos depositados no sítio florestal, mas não os teores, corroborando Cornu et al. (1998) e Scheer (2009).

Em relação ao nitrogênio, maiores valores foram observados na PI $\left(0,8 \mathrm{mg} \mathrm{L}^{-1}\right)$ do que na PE $(0,56$ $\left.\mathrm{mg} \mathrm{L}^{-1}\right)$. Em geral, os nutrientes associados às moléculas orgânicas, como o nitrogênio, são menos lixiviados, retornando ao sítio através da queda das folhas. O contrário ocorre com $\mathrm{K}$, o qual é comumente encontrado na forma iônica, portanto, movendo-se mais rapidamente pela lixiviação (EATON et al., 1973; CORTEZ, 1996; LACLAU et al., 2003). Este comportamento também foi verificado por Calil (2008) em um sistema agroflorestal no município de Candiota (RS) e por Lopes (2013) em um povoamento de Pinus taeda no município de Cambará (RS). Os maiores teores médios de potássio foram observados na PI $\left(2,06 \mathrm{mg} \mathrm{L}^{-1}\right)$ e menores na PE $\left(0,69 \mathrm{mg} \mathrm{L}^{-1}\right)$, o que resultou em maiores quantidades de K via PI com cerca de $72 \%$ do aporte total (Figura 4), o que demonstra que o elemento lavado das copas é novamente incorporado à planta através da absorção das raízes finas e volta a circular no sistema.

Tabela 2. Análise de correlação de Pearson, entre os teores e a precipitação externa (PE) em Eldorado do Sul (RS). Table 2. Pearson correlation analysis between contents and rainfall (PE) in Eldorado do Sul (RS).

\begin{tabular}{|c|c|c|c|c|c|c|c|}
\hline & $\mathbf{N}$ & $\mathrm{SO}_{4}{ }^{2-}$ & $\mathrm{Cl}^{-}$ & $\mathrm{Na}^{+}$ & $\mathrm{K}^{+}$ & $\mathrm{Ca}^{2+}$ & $\mathrm{Mg}^{2+}$ \\
\hline $\mathrm{SO}_{4}{ }^{2-}$ & $0,65^{\star *}$ & & & & & & \\
\hline $\mathrm{Cl}^{-}$ & $0,44^{* *}$ & $0,71^{* *}$ & & & & & \\
\hline $\mathrm{Na}^{+}$ & $0,33^{*}$ & $0,73^{* *}$ & $0,52^{* *}$ & & & & \\
\hline $\mathbf{K}^{+}$ & 0,39 ** & $0,69^{* *}$ & $0,59^{* *}$ & $0,66^{* *}$ & & & \\
\hline $\mathrm{Ca}^{2+}$ & 0,05 & $-0,07$ & 0,09 & $-0,14$ & $-0,14$ & & \\
\hline $\mathbf{M g}^{2+}$ & 0,15 & 0,05 & 0,14 & 0,04 & $-0,17$ & $0,53^{* *}$ & \\
\hline PE & $-0,17$ & $-0,16$ & $-0,16$ & $-0,24$ & $-0,08$ & $-0,03$ & $-0,01$ \\
\hline
\end{tabular}

${ }^{\#} \mathrm{~N}$ : Nitrogênio, o qual equivale ao somatório dos íons $\mathrm{NH}_{4}{ }^{+}, \mathrm{NO}_{3}{ }^{-}$e $\mathrm{NO}_{2}$; * * Correlação é significativa ao nível de I\%; * Correlação é significativa ao nível de $5 \% ; n=24$.

Tabela 3. Análise de correlação de Pearson entre os teores e a precipitação interna (PI) em um povoamento de eucalipto, Eldorado do Sul (RS).

Table 3. Pearson correlation analysis between contents and internal rainfall (PI) in a stand of eucalyptus, Eldorado do Sul (RS).

\begin{tabular}{c|ccccccc}
\hline & $\mathbf{N}$ & $\mathbf{S O}_{\mathbf{4}}{ }^{2-}$ & $\mathbf{C l}$ & $\mathbf{N a}^{+}$ & $\mathbf{K}^{+}$ & $\mathbf{C a}^{{ }^{+}}$ & $\mathbf{M g}^{2+}$ \\
\hline $\mathbf{S O}_{4}{ }^{2-}$ & $0,45^{* *}$ & & & & & & \\
$\mathbf{C l}^{-}$ & 0,27 & $0,49^{* *}$ & & & & & \\
$\mathbf{N a}^{+}$ & $0,34^{*}$ & $0,70^{* *}$ & $0,58^{* *}$ & & & & \\
$\mathbf{K}^{+}$ & $0,52^{* *}$ & $0,52^{\star *}$ & $0,58^{* *}$ & $0,62^{\star *}$ & & & \\
$\mathbf{C a}^{2+}$ & $-0,08$ & $-0,12$ & 0,04 & $-0,15$ & $0,34^{*}$ & & \\
$\mathbf{M g}^{2+}$ & 0,20 & 0,00 & 0,13 & 0,05 & $0,60^{* *}$ & $0,77^{* *}$ & \\
$\mathbf{P I}$ & $-0,42^{* *}$ & $-0,48^{* *}$ & $-0,51^{* *}$ & $-0,45^{* *}$ & $-0,57^{* *}$ & $-0,16$ & $-0,26$ \\
\hline
\end{tabular}

\# $\mathrm{N}$ : Nitrogênio, o qual equivale ao somatório dos íons $\mathrm{NH}_{4}^{+}, \mathrm{NO}_{\mathrm{e}}^{-}$e $\mathrm{NO}_{2} ;$; **Correlação é significativa ao nível de $1 \%$; * Correlação é significativa ao nível de $5 \% ; n=43$. 
Para o $\mathrm{Na}^{2+}$ e o $\mathrm{Cl}^{-}$, os teores médios foram de 2,16 e 2,35 $\mathrm{mg} \mathrm{L}^{-1}$, respectivamente, para a PE e de 2,94 e 3,32 $\mathrm{mg} \mathrm{L}^{-1}$ na PI. O Na ${ }^{2+}$ na água da chuva geralmente provem da lavagem de aerossóis da atmosfera (SCHEER, 2009). Porém, em alguns casos, a lavagem de exsudatos das plantas pode ter maior contribuição (CORNU et al., 1998) conforme observado na Figura 4. Os maiores teores de cloreto registrados ocorreram durante o período de menor pluviosidade (Figuras 2 e 3), Corrêa (2011) também evidenciou maiores teores de cloreto na PI $\left(0,91 \mathrm{mg} \mathrm{L}^{-1}\right)$ em relação à PE $(0,23 \mathrm{mg}$ $\mathrm{L}^{-1}$ ) em uma plantação com Eucalyptus dunni em Alegrete (RS). Os maiores teores podem estar relacionados com o maior depósito de aerossóis no dossel florestal conforme já comentado.

O sódio e o cloro (Tabelas 2 e 3), juntos, formam o cloreto de sódio que se origina da evaporação de águas superficiais que contêm o sal. A possível fonte de entrada desses elementos na área de estudo pode ser o "spray" advindo do mar, uma vez que a área se localiza à distância aproximada de 170 km em relação ao litoral. Arcova et al. (1985), Laclau et al. (2003), Carvalho Jr. (2004), Migliavacca et al. (2005) e Sá (2005) também constataram essa observação em função da proximidade das áreas de estudo em relação ao mar, considerando como fonte importante de contribuição. A proporção de cloreto na precipitação atmosférica decresce com o aumento da distância do mar, através da remoção de cristais de cloreto de sódio que atuam como condensação dos núcleos e são precipitados da atmosfera (LOSS et al., 2010). Essa observação, aliada a outros fatores, pode contribuir para o planejamento da implantação de silvicultura tendo em vista a contribuição destes elementos com a proximidade da área em relação ao mar. Segundo Tenório e Costa (2011), a concentração do sódio quando acompanha a do cloreto evidencia uma maior parte dos íons $\mathrm{Cl}^{-}$na forma salina, enquanto que a concentração de sódio quando muito menor em relação a do cloreto se caracteriza pela forma ácida, condição esta não evidenciada no presente estudo.

Prado (2008) comenta que o elemento cloro apresenta alta mobilidade na planta e participação enzimática na fotossíntese dentre outras funções, o qual não é fixado pela matéria orgânica do solo ou argilas, o que o torna facilmente lixiviado. O elemento é comumente adicionado ao sistema via fertilizantes (cloreto de potássio), pelas reservas do solo e via pluviosidade.

Além destes, os íons $\mathrm{Mg}^{2+}, \mathrm{SO}_{4}^{-} \mathrm{e} \mathrm{K}^{+}$também são considerados de origem marinha (CARVALHO JR., 2004). Mello e Motta (1987) já evidenciavam que os principais elementos químicos encontrados na água da chuva em suas formas iônicas correspondiam ao $\mathrm{Na}^{2+}, \mathrm{Ca}^{2+}, \mathrm{Mg}^{2+}, \mathrm{K}^{+}, \mathrm{Cl}^{-}, \mathrm{SO}_{4}^{2-}$, $\mathrm{NH}_{4}{ }^{+}$e $\mathrm{NO}_{3}{ }^{-}$, sendo os seis primeiros, predominantemente, de origem marinha, correlações entre esses elementos foram observadas para as duas precipitações (PE e PI) conforme as Tabelas 2 e 3. Os mesmos autores explicam que o atrito do vento com a superfície do mar faz com que borrifos d'água sejam lançados na atmosfera, formando os chamados aerossóis de origem marinha. Conforme as Tabelas 2 e 3, a correlação entre os elementos cloro, sódio e sulfato evidenciou a contribuição química de fonte marinha característica das águas pluviais. Embora a correlação entre a PI com os íons $\mathrm{Na}^{2+}, \mathrm{K}^{+}, \mathrm{Cl}^{-}$e $\mathrm{SO}_{4}{ }^{2-}$ tenha sido negativa, evidencia-se a interferência da vegetação como armazenadora desses elementos (Figura 4).

Para o sulfato, as médias observadas foram de 1,67 $\mathrm{mg} \mathrm{L}^{-1}$ (PE) e de 1,37 $\mathrm{mg} \mathrm{L}^{-1}$ (PI) o inverso foi observado em outros estudos (KOPÁČEK et al., 2011; JANÍK et al., 2011; PÉREZ SUÁREZ et al., 2008). $\mathrm{O} \mathrm{SO}_{4}{ }^{2+}$ juntamente com o $\mathrm{Ca}^{2+}$ apresentaram maiores médias para a PE do que na PI (Figura 4), o mesmo também foi observado por Caldato (2011). As menores concentrações observadas na PI podem estar relacionadas a uma possível absorção pelas copas ou a algum fenômeno de quelação que possa ter ocorrido.

Os teores médios de $\mathrm{Ca}^{2+}$ foram de 1,46 $\mathrm{mg} \mathrm{L}^{-1}$ (PE) e de 1,04 $\mathrm{mg} \mathrm{L}^{-1}$ (PI), enquanto que para o $\mathrm{Mg}^{2+}$, os teores corresponderam a 0,28 $\mathrm{mg} \mathrm{L}^{-1}$ (PE) e 0,39 $\mathrm{mg} \mathrm{L}^{-1}$ (PI). Também, verificou-se correlações entre os íons $\mathrm{Ca}^{2+}$ e o $\mathrm{Mg}^{2+}$ nas duas pluviosidades (Tabelas 2 e 3). A provável fonte de contribuição para estes elementos refere-se a poeira oriunda de estradas locais (CALIL, 2008; CALDATO, 2011), uma vez que o Ca tem origem em rochas sedimentares e metamórficas, da mesma forma que o Mg, podendo ter origem também em rochas ígneas (PRADO, 2008). Edwards (1982) e Cornu et al. (1998) sugerem que as principais fontes para o aumento $\mathrm{Ca}^{2+}$ na PI corresponde a lavagem de exsudados da vegetação e da decomposição de ramos e galhos ainda nas copas das árvores. Enquanto que, para a PE, Walling (1980) menciona a poeira dos solos da região como principal contribuinte.

As maiores quantidades de Ca foram observadas na PE, com $65 \%$ do aporte total (Figura 4), o que evidencia a precipitação como fonte importante deste elemento para o local de estudo. 


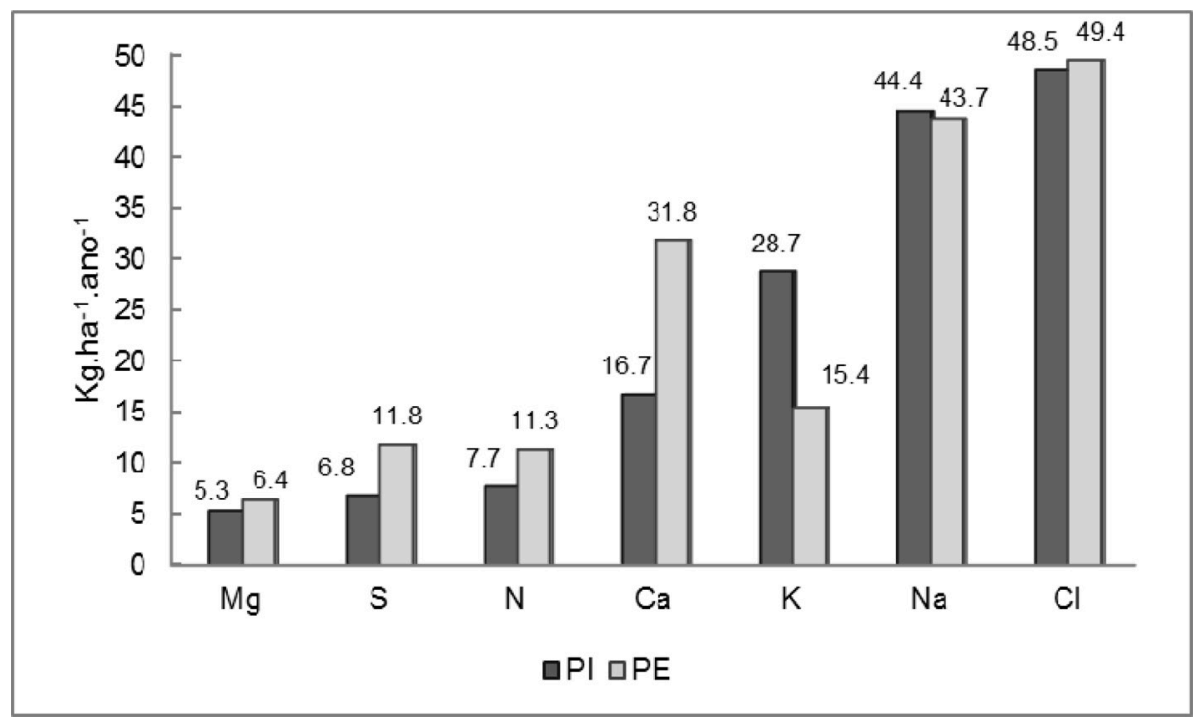

Figura 4. Quantificação média anual de macro e micronutrientes via pluviosidade externa (PE) e interna (PI) em kg ha $^{-1}$ ano $^{-1}$.

Figure 4. Quantification of the annual mean of macro and micronutrients in the rainfall (PE) and internal rainfall (PI) in $\mathrm{kg} \mathrm{ha}^{-1} \cdot \mathrm{yr}^{-1}$.

Do total aportado, a PI contribuiu com $43 ; 66 ; 35 ; 47 ; 37 ; 51$ e $50 \%$, respectivamente, para o N, $\mathrm{K}, \mathrm{Ca}, \mathrm{Mg}$, S, Na e Cl. Esses dados sugerem que o volume precipitado influenciou na lavagem/lixiviação dos nutrientes no dossel florestal. Dezzeo e Chacón (2006) investigando três tipologias florestais na Venezuela verificaram um aumento dos teores de cátions na PI em torno de 80, 31 e 15\%, respectivamente, para os elementos $\mathrm{K}, \mathrm{Ca} \mathrm{e} \mathrm{Mg}$, similar à magnitude observada no presente estudo.

Balieiro et al. (2007), em um estudo com eucalipto no município de Seropédica (RJ), verificou um aporte maior de nutrientes na PI em relação à PE de 63,36; 7,62; 5,68 e 23,47 kg ha'-1 para os elementos $\mathrm{K}, \mathrm{Ca}$, Mg e Na, respectivamente. Da mesma forma, maiores quantidades foram observadas por Caldato (2011) na PI, sendo de 28,06 ( $\left.\mathrm{Ca}^{2+}\right) ; 12,16(\mathrm{Cl}) ; 4,05\left(\mathrm{Na}^{2+}\right)$ e 54,09 $\mathrm{kg} \mathrm{ha}^{-1} \mathrm{ano}^{-1}$ $\left(\mathrm{K}^{+}\right)$em plantio do gênero Pinus. Scheer (2009) observou um aporte anual de $52\left(\mathrm{~K}^{+}\right), 5 \mathrm{de}\left(\mathrm{Ca}^{2+}\right)$ e 2,9 $\mathrm{kg} \mathrm{ha}^{-1}\left(\mathrm{Mg}^{2+}\right)$ em área de capoeira e $29\left(\mathrm{~K}^{+}\right)$; 4,2 $\left(\mathrm{Ca}^{2+}\right) ; 2\left(\mathrm{Mg}^{2+}\right)$ e $92 \mathrm{~kg} \mathrm{ha}^{-1}\left(\mathrm{Na}^{2+}\right)$ em área de Floresta Ombrófila Densa. Com base nesses resultados, verifica-se que tais variações observadas indicam a importância desses estudos na compreensão do ciclo geoquímico para os diversos ecossistemas, espécies, diferentes localidades e sob diferentes graus de interferência antrópica. Dessa forma, verifica-se que o grau de modificação na caracterização da água da chuva varia em função desses fatores, do tempo, da disponibilidade de aerossóis, exsudatos das plantas e da intensidade das pluviosidades.

É importante ressaltar que nem toda a quantidade de nutrientes resultante da PI representa, todavia, adição nova ao solo. Uma vez que parte desses nutrientes provém do ciclo biogeoquímico local, o qual resulta da retirada, pela água, de metabólitos lábeis das folhas das árvores, devolvendoos ao solo.

\section{CONCLUSÕES}

A concentração dos elementos químicos na água da chuva variou conforme os volumes de pluviosidade, evidenciando-se uma correlação inversa dos teores com o aumento da precipitação.

Os íons potássio, sulfato, cloreto, sódio e os que compõem o elemento nitrogênio mostraram uma correlação significativa com a pluviosidade interna $(\mathrm{p}<0,01)$.

A quantidade de elementos químicos carreada via pluviosidade evidenciou a importância que esta fonte de entrada nutricional representa para a manutenção da fertilidade do solo e, consequentemente, para a sustentabilidade da atividade de silvicultura com o Eucalyptus urophylla $\mathrm{x}$ Eucalyptus globulus subsp. maidenii. 


\section{REFERÊNCIAS BIBLIOGRÁFICAS}

ALLEN, S. E.; CARLISLE, A.; WHITE, E. J.; EVANS, C. C. The plant nutrient content of rainwater. Journal of Ecology, Oxford, v. 56, n. 2, p. 497-504, 1968.

APHA - AMERICAN PUBLIC HEALTH ASSOCIATION. Standard methods for the examination of water and wastewater. 19.ed. Washington, 1998.

ARCOVA, F. C. S.; CICCO, V. Fluxo de nutrientes através da precipitação, precipitação interna e escoamento pelo tronco em floresta natural secundária do Parque Estadual da Serra do Mar - Núcleo Cunha-SP. Boletim Técnico Instituto Florestal. São Paulo, v. 41, n. 1, p. 37-58, 1987.

ARCOVA, F. C. S.; CICCO, V.; LIMA, W. P. Balanço dos nutrientes $\mathrm{Ca}^{2+}, \mathrm{Mg}^{2+}, \mathrm{Na}^{+1}, \mathrm{~K}^{+1}$ e $\mathrm{NO}_{3}^{-}$em bacia hidrográfica experimental com vegetação natural do Parque Estadual da Serra do Mar - Núcleo Cunha - SP. IPEF, Piracicaba, n. 31, p. 61-67, dez. 1985.

ARCOVA, F. C. S.; CICCO, V.; SHIMOMICHI, P. Y. Qualidade da água e dinâmica dos nutrientes em bacia hidrológica recoberta por floresta de mata atlântica. Revista do Instituto Florestal, São Paulo, v. 5, n.1, p. $1-20,1993$.

ARTAXO, P.; OLIVEIRA, P. H.; LARA, L. L.; PAULIQUEVIS, T. M.; RIZZO, L. V.; PIRES JR., C.; PAIXÃO, M. A.; LONGO, K. M.; FREITAS, S.; CORREIA, A. L. Efeitos climáticos de partículas de aerossóis biogênicos e emitidos em queimadas na Amazônia. Revista Brasileira de Meteorologia, Rio de Janeiro, v. 21, n. 3a, 168-22, 2006.

BALIEIRO F. C.; FRANCO, A. A.; FONTES, R. L. F.; DIAS, L. E.; CAMPELLO, E. F. C.; FARIA, S. M. Evaluation of the throughfall and stemflow nutrient contents in mixed and pure plantations of Acacia mangium, Pseudosamenea guachapele and Eucalyptus grandis. Revista Árvore, Viçosa, v. 31, n. 2, p. 339-346, 2007.

BELLOTE, A. F. G.; DEDECEK, R. A.; SILVA, H. D. Nutrientes minerais, biomassa e deposição de serapilheira em plantios de Eucalyptus com diferentes sistemas de manejo de resíduos florestais. Pesquisa Florestal Brasileira, Colombo, n. 56, p. 31-41, 2008.

CALDATO, S. L. Ciclagem biogeoquímica dos nutrientes em uma plantação de Pinus taeda L. no nordeste Argentino. 2011. 106 p. Tese (Doutorado em Silvicultura) - Universidade Federal de Santa Maria, Santa Maria, 2011.

CALIL, F. N. Aspectos nutricionais de um sistema agroflorestal com Eucalipto no sul do Rio Grande do Sul, Brasil. 2008. 144 p. Tese (Doutorado em Engenharia Florestal) - Universidade Federal de Santa Maria, Santa Maria, 2008.

CARVALHO JR., V. N. Deposição atmosférica e composição química da água de chuva. Revista Tecnológica, Fortaleza, v. 25, n. 2, p. 61-71, dez. 2004.

CASARTELLI, M. R.; MIRLEAN, N.; PERALBA, M. C.; BARRIONUEVO, S.; GÓMEZ-REY, M. X.; MADEIRA, M. An assessment of the chemical composition of precipitation and throughfall in rural-industrial gradient in wet subtropics (southern Brazil). Environmental Monitoring and Assessment, v. 144, n. 1-3, p. 105-116, 2008.

CORNU, S.; AMBROSI, J. P.; LUCAS, Y.; DESJARDINS, T. Origin and behavior of dissolved chlrorine and sodium in Brazilian Rainforest. Water Research, London, v. 32, n. 4, p. 1151-1161, 1998.

CORRÊA, R. S. Ciclagem de nutrientes em Eucalyptus dunnii estabelecido no bioma Pampa. 2011. 99 p. Tese (Doutorado em Engenharia Florestal) - Universidade Federal de Santa Maria, Santa Maria, 2011. 
Valente et al. - Quantificação de nutrientes na precipitação em um plantio de

Eucalyptus urophylla x Eucalyptus globulus subsp. maidenii, Eldorado do Sul, RS

CORTEZ, N. R. S. Compartimentos e ciclo de nutrientes em plantações de Eucalyptus globulus Labill Ssp. globulus e Pinus pinaster Aiton. 1996. 317 p. Thesis (Ph.D.) - Instituto Superior de Agronomia, Lisboa, 317 p. 1996.

CURI, N., MARQUES, J. J., MENEZES, M. D. Descrição morfológica do perfil do solo da área experimental. [mensagem pessoal]. Mensagem recebida por: . Acesso em 26 ago. 2009.

DEZZEO, N.; CHACÓN, N. Nutrient fluxes in incident rainfall, throughfall, and stemflow in adjacent primary and secondary forests of the Gran Sabana, southern Venezuela. Forest Ecology and Management, Amsterdam, v. 234, n. 1-3, p. 218-226, 2006.

EATON, J. S.; LIKENS, G. E.; BORMANN, H. Throughfall and stemflow chemistry in a northern hardwood forest. Journal of Ecology, Oxford, v. 61, n. 2, p. 495-508, 1973.

EDWARDS, P. J. Studies of mineral cycling in a montane rain forest in New Guinea. V. Rates of cycling in througfall and litter fall. Journal of Ecology, Oxford, v. 70, n. 3, p. 807-827, 1982.

FREITAS, S. R.; SILVA DIAS, M. A. F.; SILVA, DIAS, P. L.; LONGO, K. M.; ARTAXO, P.; ANDRAE, M. O.; FISCHER, H. A convective kinematic trajectory technique for low resolution atmospheric models. Journal of Geophysical Research, Washington, v. 105, n. D19, p. 24.375-24.386, 2000.

HAAG, H. P. Chuvas ácidas. Campinas: Fundação Cargil, 1985. 77 p.

KOPÁČEK, J.; TUREK, J.; HEJZLAR, J.; PORCAL, P. Bulk deposition and throughfall fluxes of elements in the Bohemian Forest (Central Europe) from 1998 to 2009. Boreal Environment Research, v. 16, n. 6, p. 495-508. 2011.

JANÍK, R.; BUBLINEC, E.; DUBOVÁ, M. Impact of regeneration cutting on sulphate concentration and amount in throughfall water in conditions of submontane beech forests in the Western Carpathians Mts. Folia Oecologica. v. 38. n. 2. p. 156-161. 2011.

JUKNYS, R., ZALTAUSKAITE, J., STAKENAS, V. Ion Fluxes with Bulk and Throughfall Deposition along an Urban - Suburban - Rural Gradient. Water, Air, and Soil Pollution, v. 178, n. 1, p. 363-372, 2007.

LACLAU, J. P.; RANGER, J.; BOUILLET, J. P.; NZILA, J. D.; DELEPORTE, P. Nutrient cycling in a clonal stand of Eucalyptus and an adjacent savanna ecosystem in Congo 1. Chemical composition of rainfall, throughfall and stemflow solutions. Forest Ecology and Management, Amsterdam, v. 176, n. 1-3, p. 105-119, 2003.

LIMA, W. P. Entrada de nutrientes pela chuva e pela lixiviação das copas em florestas homogêneas de pinheiros tropicais e em vegetação natural de Cerradão. Acta Limnologica Brasiliensis, São Carlos, v. 1, p. 59-62, 1986.

LIMA, W. P. Ação das chuvas no ciclo biogeoquimico de nutrientes em plantações de pinheiros tropicais e em cerradão. IPEF, Piracicaba, n. 30, p.13-17, 1985.

LIU, W.; FOX, J. E. D.; XU, Z. Nutrient fluxes in bulk precipitation, throughfall and stemflow in montane subtropical moist forest on Ailao Mountains in Yunnan, south-west China. Journal of Tropical Ecology, Cambridge, v. 18, n. 4, p. 527-548, 2002.

LOPES, M. I. M. S. Fluxo de água, balanço químico e alterações no solo da Floresta Atlântica atingida pela poluição aérea de Cubatão, SP, Brasil. 2001. 188 p. Tese (Doutorado em Ciências, na Área de Ecologia) Universidade de São Paulo, São Paulo, 2001.

LOPES, V. G. Dinâmica nutricional em um povoamento de Pinus taeda l., Rio Grande do Sul, Brasil. 2013. 138 p. Tese (Doutorado em Engenharia Florestal) - Universidade Federal de Santa Maria, Santa Maria, 2013. 
LOSS, A.; ROSSI, C. Q.; FRANCELINO, M. R. A origem da salinidade no Continente Antártico. Revista de Ciências Agrárias. Lisboa, v. 33 n. 2, dez. 2010.

MALUF, J. R. T. Nova classificação climática do Estado do Rio Grande do Sul. Revista Brasileira de Agrometeorologia, Santa Maria, v. 8, n. 1, p. 141-150, 2000.

MEGURO, M.; VINUEZA, G. N.; DELITTI, W. B. C. Ciclagem de nutrientes minerais na mata mesófila secundária - São Paulo. II - O papel da precipitação na importação de potássio e fósforo. Boletim de Botânica, São Paulo, v. 7, p. 61-67, 1979.

MELLO, W. Z.; MOTTA, J. S. T. Acidez na chuva. Ciência Hoje, Rio de Janeiro, v. 6, n. 34, p. 40-43. 1987.

MIGLIAVACCA, D. M.; TEIXEIRA, E. C.; MACHADO, A. C. M.; PIRES, M. R. Composição química da precipitação atmosférica no sul do Brasil - Estudo preliminar. Revista Química Nova, São Paulo, v. 28, n. 3, p. 371-379, 2005.

PEHL, C. E.; RAY, K. F. Atmospheric nutrient inputs to three forest types in east Texas. Forest Ecology and Management, Amsterdam, v. 7, n. 1, p. 11-8, 1983.

PÉREZ SUÁREZ, M.; FENN, M. E.; ALCALÁ, V. M. C.; ALDRETE, A. The effects of canopy cover on throughfall and soil chemistry in two forest sites in the México City air basin. Atmósfera, México, v. 21, n. 1, p. 83-100, 2008.

PRADO, R. M. Nutrição de plantas. São Paulo: Editora UNESP, 2008. 407 p.

SÁ, S. S. Caracterização geoquímica das precipitações atmosféricas do município de Rio Grande, RS. 2005. 91 p. Dissertação (Mestrado em oceanografia física, química e geológica) - Fundação Universidade Federal do Rio Grande, Rio Grande, 2005.

SCHEER, M. B. Ciclagem de nutrientes em um trecho de Floresta Ombrófila Densa Aluvial em regeneração, Guaraqueçaba, PR. Curitiba, 2006. 155 p. Dissertação (Mestrado em Engenharia Florestal) - Universidade Federal do Paraná, Curitiba, 2006.

SCHEER, M. B. Fluxo de nutrientes pela precipitação pluviométrica em dois trechos de Floresta Ombrófila Densa em Guaraqueçaba, Paraná. Curitiba, PR, Floresta, Curitiba, v. 39, n. 1, p. 117-130, jan./mar. 2009.

SILVA, W.; DERECZYNSKI, C.; CHOU, S. C.; CAVALCANTI, I. Future Changes in Temperature and Precipitation Extremes in the State of Rio de Janeiro (Brazil). American Journal of Climate Change, v. 3, n. 4, p. 353-365, 2014.

SOUZA, L. C.; MARQUES, R. Fluxo de nutrientes em floresta ombrófila densa das terras baixas no litoral do Paraná. Floresta, Curitiba, v. 40, n. 1, p. 125-136, jan./mar. 2010.

TENÓRIO, A. M.; COSTA, W. Um estudo da deposição de íons e matéria orgânica por meio das precipitações pluviométricas. Acta Scientiarum Technology, Maringá, v. 33, n. 4, p. 457-463, 2011.

WALLING, D. E. Water in the Catchment Ecosystems. In: Water Quality in catchment Ecosystems. Chichester: John Wiley, 1980. p.1-47.

ZHANG, G.; ZENG, G. M.; HUANG, G. H.; JIANG, Y. M.; YAO, J. M.; DU, C. Y.; JIANG, R.; ZHANG, C. Deposition pattern of precipitation and throughfall in a subtropical evergreen in south-central China, The Japanese Forest Society and Springer, v. 11, n. 6, p. 389-396, Jun. 2006.

Recebido em 19/08/2014

Aceito para publicação em 15/10/2015

Sci. For., Piracicaba, v. 44, n. 109, p. 249-259, mar. 2016 DOI: dx.doi.org/10.18671/scifor.v44n109.24 
